\title{
BMJ Open The prevalence of type 2 diabetes mellitus among older people in Africa: a systematic review study protocol
}

\author{
Mahmoud Werfalli, ${ }^{1,2,3}$ Alfred Musekiwa, ${ }^{4}$ Mark E Engel, ${ }^{2}$ lan Ross, ${ }^{2,3}$ \\ Andre P Kengne, ${ }^{4,5}$ Naomi S Levitt ${ }^{1,2,3}$
}

To cite: Werfalli $\mathrm{M}$, Musekiwa A, Engel ME, et al. The prevalence of type 2 diabetes mellitus among older people in Africa: a systematic review study protocol. BMJ Open 2014;4: e004747. doi:10.1136/ bmjopen-2013-004747

- Prepublication history and additional material is available. To view please visit the journal (http://dx.doi.org/ 10.1136/bmjopen-2013004747).

Received 23 December 2013 Revised 11 April 2014 Accepted 16 May 2014

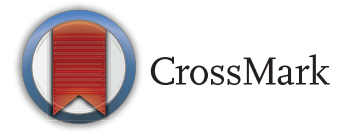

For numbered affiliations see end of article.

Correspondence to Dr Naomi Levitt; Naomi.levitt@uct.ac.za

\section{ABSTRACT}

Introduction: The number of people with diabetes in Africa is projected to increase substantially in the next two decades, due to factors including rapid urbanisation, adoption of unhealthy diets and exercise patterns and the ageing of the population. There are currently uncertainties regarding the incidence, prevalence and management patterns of diabetes in older people across the diversity of African countries. We wish to perform a systematic review to determine the prevalence of type 2 diabetes in Africa in the older individual, over the age of 55 years, reported in studies from 2000 to 2013.

Methods and analyses: A comprehensive literature search among a number of databases will be undertaken, using an African search filter to identify diabetes prevalence studies that were published from 2000 to 2013. Full copies of articles identified by the search, and considered to meet the inclusion criteria, will be obtained for data extraction and synthesis. Statistical analysis of the primary measures, fasting plasma glucose (FPG) and oral glucose tolerance test will include two steps: (1) identification of data sources and documenting estimates and (2) application of the random-effects meta-analysis model to aggregate prevalence estimates and account for between study variability in calculating the overall pooled estimates and $95 \% \mathrm{Cl}$ for diabetes prevalence. Heterogeneity will be evaluated using the $\mathrm{I}^{2}$ statistic to determine the extent of variation in effect estimates that is due to heterogeneity rather than chance. This systematic review will be reported according to the Preferred Reporting Items for Systematic reviews and MetaAnalyses (PRISMA).

Ethics and dissemination: Ethics is not required for this study, given that this is a protocol for a systematic review, which utilises published data. The findings of this study will be widely disseminated through peerreviewed publications and conference presentations.

\section{INTRODUCTION}

The most recent International Diabetes Federation (IDF) estimates from 2013 are that $8.3 \%$ of adults that is, 382 million people worldwide have diabetes. This number has doubled over the past 20 years, and notably $80 \%$ of people with diabetes live in low and middle income countries (LMIC). ${ }^{1}$ Diabetes already contributes significantly to morbidity and mortality in Africa. The highest global age-specific mortality rate is recorded in this continent. ${ }^{2-6}$ All countries in Africa fall into the LMIC category, and predominantly the low income category. The rise in the number of individuals with type 2 diabetes in Africa, similar to LMIC has been attributed to ageing of the population and relatively rapidly changing environmental factors. ${ }^{1}$ These include urbanisation, the adoption of health behaviours favouring sedentariness and unhealthy eating patterns. While unhealthy behaviour patterns and obesity are potentially modifiable, ageing, one of the major drivers for diabetes, is not. ${ }^{7}$ In 2013, the majority of individuals with diabetes in Africa were reported to be under 60 years of age with the highest proportion $(43.2 \%)$ in people aged $40-59$ years. ${ }^{7}$ The relatively small proportion of individuals aged $60-79$ years in the region is likely to account for the estimate that only $18.8 \%$ of patients with diabetes fall in this age group. ${ }^{1}$

Africa is often referred to as the youngest continent in terms of age structure. This may contribute to the current relatively low prioritisation of ageing issues in national policies. ${ }^{8}$ Yet the annual growth rate of older persons in Africa has been estimated at $3.1 \%$ between 2007 and 2015, and 3.3\% between 2015 and 2050, greater than the global average. In this context, it is concerning that there will be approximately 64.5 million Africans aged $\geq 55$ years in 2015 , and more than 103 million and 205 million in 2030 and 2050, respectively. ${ }^{6}$ Indeed it has been predicted that the diabetes peak in Africa is expected to be in the oldest individual by 2035. ${ }^{1}$ We therefore wish to perform a 
systematic review to determine the prevalence of type 2 diabetes in Africa in older individuals over the age of 55 years, reported in studies from 2000 to 2013 with a view to providing accurate data for monitoring future trends. The data will also be of value in informing health policy makers of the extent of the burden of diabetes in an under researched group whose healthcare needs may differ from those in younger adults.

\section{OBJECTIVES}

To conduct a systematic review and meta-analysis of studies assessing the prevalence of type 2 diabetes among older people in African countries.

\section{Review question}

This systematic review will be guided by the following research question:

What is the prevalence of type 2 diabetes in older persons aged 55 years and older in African countries as reported in studies from 2000 to 2013?

\section{Criteria for considering studies for review}

Inclusion criteria

1. Studies describing the prevalence of type 2 diabetes among older adults, resident in countries belonging to the African continent, in the geographic regions of sub-Saharan and North Africa diagnosed with type 2 diabetes from all ethnicities, socioeconomic and educational backgrounds. Participants should be described as older adults or a minimum of $70 \%$ of participants should be within the age groups of $55-64,65-74$ or $75+$ years.

2. Population-based studies, cross-sectional studies of type 2 diabetes. For the purpose of this review, the diagnosis of diabetes should be made by a physician or defined by available measured fasting plasma glucose (FPG), oral glucose tolerance test (OGTT) or self-reported, according to WHO criteria. ${ }^{9}$

We will consider published articles and unpublished studies reported after 1 January 2000, given that the current criteria for the diagnosis of diabetes have been widely accepted since 1998. Articles published in any language, with full English abstracts will be eligible for inclusion.

Exclusion criteria

1. Studies which include a mixed group of type 1 and type 2 participants, or that do not clearly define the type of diabetes as being type 2, will be excluded.

2. Studies confined to subgroups of patients with type 2 diabetes (with any complication of diabetes mellitus (DM) eg: myocardial infarction, eye, kidney or other microvascular or macrovascular complications).

3. Studies that do not include a representative sample of older people aged 55 years or older.

4. Narrative reviews, opinion pieces, letters or any other publications lacking primary data and/or explicit method descriptions.
5. Duplicate publications of the same material. When the study has been published in more than one journal/conference; the most complete recent version will be used.

6. The studies that had low-quality scores (equal to or below 5 ) in the assessment of risk of bias.

\section{Search strategy for identification of relevant studies}

The search strategy will comprise two stages:

\section{Bibliographic databases}

A. A comprehensive and sensitive search strategy will be undertaken using an African search filter developed by Siegfried ${ }^{10}$ to identify prevalence studies conducted from 2000 to the African filter comprising country names from the continent as well as truncated terms such as 'north* Africa' to ensure that records indexed using regional, rather than countryspecific, terms are also retrieved. Database subject headings (MeSH in PubMed/MEDLINE, CINHAL and Google Scholar) will be combined with a range of text words (see online supplementary appendix 1). African country names are included in English and languages relevant to the country, for example, 'Ivory Coast' and 'Cote d'Ivoire'. Where country names have changed over time both names are included, for example, 'Democratic Republic of Congo 'and 'Zaire'. 11

B. Publications of identified key authors will be examined by citation searches on the IDF and WHO websites for example, STEPS surveys studies in Africa as well as on the ISI Web of Knowledge platform. A bibliographic software programme for managing the references and documenting the study selection process will be used for this review. An expert librarian will help in designing the search strategy framework and implementing the appropriate bibliographic software programme (for the detailed search strategy, see online supplementary appendix 2.)

\section{Selecting studies for inclusion}

Full copies of articles identified by the search, and considered to meet the inclusion criteria, based on the title and abstract will be obtained for data synthesis. Initially, studies will be screened using predefined inclusion and exclusion criteria. Two reviewers will apply the criteria independently to the results of the searches, based first on titles and abstracts only. Studies will then be either (1) excluded, (2) included or (3) marked as 'pending' if the reviewer is unsure about their inclusion. The two independent reviews will be compared and contradictory judgments or 'pending' will be temporarily 'included', and moved to the next phase of review of full texts. Once full texts have been retrieved, two reviewers will independently apply inclusion and exclusion criteria, based on quick assessments of the full texts. Disagreements in reviewer selections will be resolved at a meeting between reviewers prior to selected articles 
being retrieved. A flow chart will be produced to facilitate transparency of the process.

\section{Quality appraisal of included studies}

A quality assessment tool, based on guidelines for evaluating prevalence studies as suggested by Hoy et $a l^{12}$ has been developed (table 1). This will be applied to screened full-text articles in order to code eligibility decisions and to assess study quality and agreement between investigators. Assessment of bias is built into the quality scoring scale. We plan to evaluate risk of selection and attrition bias using the Cochrane guidelines as set out in Review Manager V.5.2 (http://ims.cochrane. org/RevMan). This will inform the feasibility of and selection of studies for a pooled analysis. Any disagreements will be resolved by discussion and consensus in consultation with the third author to resolve persistent inconsistencies.

\section{Data extraction and management}

Following assessment of methodological quality, two reviewers will extract data onto a purpose-designed data extraction form and independently summarise what they consider to be the most important results from each study. These summaries will be compared and any differences of opinion will be resolved by discussion and consultation with a third reviewer. Any further calculations on study data considered necessary, will be conducted by the first reviewer and checked by the second reviewer. Study characteristics including country where study was conducted, year of publication, journal, language of publication, study population, age range, response rate, study design, criteria for sample selection and sample size, outcome(s) measured, diagnostic criteria, results and notes/comments will be presented in tables (see online supplementary appendix 3). We are anticipating that some eligible studies will not have prevalence data reported for the specific age range (ie, $\geq 55$ years). We will contact the corresponding authors of these studies and request the age-specific prevalence and any other missing information, deemed to be relevant.

\section{Data synthesis including assessment of heterogeneity}

Our statistical analysis of the primary measures, FPG and OGTT will include two steps: (1) identification of data sources and documenting estimates and (2) application of the random-effects meta-analysis model to aggregate prevalence estimates and account for between study variability in calculating the overall pooled estimates and 95\% CI for diabetes prevalence. We will derive SEs where studies have provided the corresponding numerator and denominator for diabetes prevalence estimates. We will consider non-overlapping CIs as an indication of statistically significant differences. Prevalence of type 2 DM from different studies will be pooled in a meta-analysis using (STATA V.12 statistical software). Heterogeneity will be assessed by inspecting forest plots initially, then through the Cochran's $\chi^{2}$ test using a $10 \%$ level of significance cut-off (due to the low power of the test), and the $\mathrm{I}^{2}$ statistic where values of $25 \%, 50 \%$ and $75 \%$ reflect low, medium and high heterogeneity, respectively. ${ }^{13}$ Where heterogeneity is statistically significant, subgroup analysis, using the following variables: age group, sex, setting for example, urban/rural geographical region for example, northern/southern, western/eastern, as well as sensitivity analyses will be conducted to determine the potential sources of heterogeneity.

Furthermore a sensitivity analysis will be performed to evaluate how excluding studies that did not meet each quality criterion would affect our overall estimate. Another sensitivity analysis will be conducted to find out how our results would change if only high-quality studies were considered. If the identified studies are of substantial heterogeneity and where statistical pooling is not possible, the findings will be presented in narrative form

Table 1 Quality assessment criteria for prevalence studies ${ }^{12}$

External validity

1. Was the study's target population a close representation of the national population in relation to relevant (1 point) variables?

2. Was the sampling frame a true or close representation of the target population?

3. Was some form of random selection used to select the sample, OR was a census undertaken?

4. Was the likelihood of non-response bias minimal?

(1 point)

(1 point)

(1 point)

Total (4 points)

Internal validity

1. Were data collected directly from the participants (as opposed to a proxy)?

2. Was an acceptable case definition used in the study?

3. Was the study instrument that measured the parameter of interest shown to have validity and reliability?

4. Was the same mode of data collection used for all participants? (1 point)

5. Was the length of the shortest prevalence period for the parameter of interest appropriate?

6. Were the numerator(s) and denominator(s) for the parameter of interest appropriate?
(1 point)

(1 point)

(1 point)

(1 point)

(1 point)

(1 point)

Total (6 points) 
including tables and figures to aid in data presentation where appropriate. The narrative will be written by the two reviewers and then checked independently by the other reviewers, any disagreements will be decided by all reviewers.

\section{ASSESSMENT OF REPORTING BIASES}

Symmetry of funnel plots will be used to assess for publication or selective reporting bias.

\section{REPORTING OF THIS REVIEW}

This systematic review will be reported according to the Preferred Reporting Items for Systematic reviews and Meta-Analyses (PRISMA) Statement. ${ }^{13}$ A reporting guideline for systematic reviews of healthcare intervention will include a PRISMA checklist. Where necessary, we will adapt the reporting to ensure that all items relevant to this review are included in the report.

\section{ETHICS AND DISSEMINATION}

Given that this is a protocol for a systematic review, which utilises published data, ethics is not required for this study. The findings of this study will be widely disseminated through peer-reviewed publications, conference presentations and submitted to relevant authorities in national departments of health. Updates of the review will be conducted to inform and guide healthcare practice.

\section{Author affiliations \\ ${ }^{1}$ Chronic Disease Initiative for Africa (CDIA), Cape Town, South Africa \\ ${ }^{2}$ Department of Medicine, University of Cape Town, Cape Town, South Africa \\ ${ }^{3}$ Division of Diabetic Medicine and Endocrinology, Department of Medicine, University of Cape Town, Cape Town, South Africa \\ ${ }^{4}$ Faculty of Medicine and Health Sciences, Centre for Evidence Based Health Care, Stellenbosch University, Cape Town, South Africa \\ ${ }^{5}$ Non-Communicable Diseases Research Unit, South African Medical Research Council \& University of Cape Town, Cape Town, South Africa}

Acknowledgements The authors acknowledge Dr Taryn Young, Evidence-based health care Centre, and University of Stellenbosch, who provided guidance for designing the protocol. Ms Tamzyn Suliaman, UCT Libraries, Health Sciences, Information Services Librarian, provided technical support and assisted in the planning of the search strategy and reference management. Finally, they gratefully acknowledge support of the
Evidence-Based Medicine Research Support Unit, Faculty of Health Sciences at University of Cape Town.

Contributors All authors conceived the study and were responsible for designing the protocol. MW and AM co-drafted the protocol manuscript. MEE and NSL provided critical guidance on the analysis and overall direction of the study. IR and APK revised it for methodological and clinical content. All authors critically revised successive drafts of the manuscripts and approved the final version.

Competing interests None.

Provenance and peer review Not commissioned; externally peer reviewed.

Open Access This is an Open Access article distributed in accordance with the Creative Commons Attribution Non Commercial (CC BY-NC 3.0) license, which permits others to distribute, remix, adapt, build upon this work noncommercially, and license their derivative works on different terms, provided the original work is properly cited and the use is non-commercial. See: http:// creativecommons.org/licenses/by-nc/3.0/

\section{REFERENCES}

1. Guariguata L, Whiting D, Hambleton I, et al. Global estimates of diabetes prevalence for 2013 and projections for 2035 for the IDF Diabetes Atlas. Diabetes Res Clin Pract 2014;103:137-49.

2. Alli F, Maharaj P. The health situation of older people in Africa. Aging and Health in Africa Springer, 2013:53-89.

3. Steyn K, Damasceno A. Lifestyle and related risk factors for chronic diseases. Disease and mortality in sub-Saharan Africa. 2006;2.

4. Guariguata L, Whiting D, Weil C, et al. The International Diabetes Federation diabetes atlas methodology for estimating global and national prevalence of diabetes in adults. Diabetes Res Clin Pract 2011;94:322-3.

5. Levitt NS. Diabetes in Africa: epidemiology, management and healthcare challenges. Heart 2008;94:1376-82.

6. Naidoo N, Abdullah S, Bawah A, et al. Ageing and adult health status in eight lower-income countries: the INDEPTH WHO-SAGE collaboration. Global Health Action, 2010:11.

7. Sobngwi E, Mauvais-Jarvis F, Vexiau P, et al. Diabetes in Africans. Diabetes Metab 2001;27:628-34.

8. Dalal S, Beunza JJ, Volmink J, et al. Non-communicable diseases in sub-Saharan Africa: what we know now. Int J Epidemiol 2011;40:885-901.

9. World Health Organization. Definition and diagnosis of diabetes mellitus and intermediate hyperglycaemia: report of a WHO/IDF consultation. Geneva: World Health Organization, 2006:1-50.

10. Eisinga A, Siegfried N, Clarke M. The sensitivity and precision of search terms in Phases I, II and III of the Cochrane Highly Sensitive Search Strategy for identifying reports of randomized trials in Medline in a specific area of health care-HIV/AIDS prevention and treatment interventions. Health Info \& Libr J 2007;24:103-9.

11. Pienaar E, Grobler L, Busgeeth $\mathrm{K}$, et al. Developing a geographic search filter to identify randomised controlled trials in Africa: finding the optimal balance between sensitivity and precision. Health Info Libr J 2011;28:210-15.

12. Hoy $D$, Brooks $P$, Woolf $A$, et al. Assessing risk of bias in prevalence studies: modification of an existing tool and evidence of interrater agreement. J Clin Epidemiol 2012;65:934-9.

13. Higgins JP, Green S, Collaboration C. Cochrane handbook for systematic reviews of interventions. Wiley Online Library, 2008. 